\title{
3D Depth of Field Acquisition Based on OpenCV
}

\author{
Feng $\mathrm{Yu}^{1, \mathrm{a}}$, Junfeng Shuai ${ }^{2, \mathrm{~b}}$,Baolin $\mathrm{Yin}^{2, \mathrm{c}}$ and Xiaoming Feng ${ }^{2, \mathrm{~d}}$ \\ School of Mechanical Engineering, Jiamusi University, Jiamusi154007, China \\ a7792222180@qq.com, b454415020@qq.com, cyinblin@163.com, d1159527506@qq.com \\ Corresponding author: Junfeng Shuai
}

Keywords: Computer vision, OpenCV, camera calibration, stereo matching.

\begin{abstract}
D reconstruction model of OpenCV is established based on the 3D reconstruction technique of computer vision. The camera calibration, stereo calibration, image correction, stereo matching are used for acquiring disparity map, the 3D coordinates of the target points are obtained by the disparity map, the interface functions of corresponding algorithm provided by OpenCV are used for completing the above steps, the 3D points cloud display are carried out by Matlab. It has higher arithmetic speed and accuracy of point position with good cross platform portability, which can meet the needs of a variety of computer vision systems.
\end{abstract}

\section{Introduction}

3D reconstruction technology is a hot and difficult field of computer vision, artificial intelligence, and virtual reality and so on. It is one of the major challenges in the basic and applied research. Image based 3D reconstruction is an important research branch of image processing, and it is widely used in the detection and observation as the basis of virtual reality and scientific visualization. A complete 3D reconstruction system can be divided into image acquisition, camera calibration, stereo calibration, image correction, stereo matching and post-processing and so on. Among the accurate calibration of inside and outside parameters of camera and stereo matching is the most important and difficult problem in 3D reconstruction.

OpenCV is developed by Intel Corporation, it is a library consisting of C functions and C++ classes to achieve the commonly used image processing and computer vision algorithm. The good interface is provided for users by camera calibration module of OpenCV, and it can be used in platforms of windows, Linux, Mac OS, the development efficiency and execution speed can be improved with good portability of cross platform, so it can be well used in practical engineering.

\section{The Basic Principle of 3D Depth of Field Acquisition}

\subsection{Image Acquisition.}

Image acquisition is the basis for stereo vision, it mainly depends on the occasions and purposes of application, but also the factors such as viewpoint, light, camera performance and scenery features should be considered to facilitate the 3D calculation. The left and right images of the target object are obtained by fixing the left and right camera at the same time shooting target objects. The selected camera resolution is $640 * 480$, the pixel is 3 million, and the sampling frequency is 15 frame / sec.

\subsection{Camera Calibration.}

One of the basic tasks of computer vision is the geometric and structural information of objects in $3 \mathrm{D}$ space is calculated based on the 2D image information from the camera, the purpose of reconstruction and identification of objects is achieved, the mapping relationship between a point on the surface of space object and corresponding points in the image is decided by the geometric model of camera imaging. The imaging models, the position of the camera and attribute parameters are established by camera calibration to determine the mapping relationship between object points and its image points in the spatial coordinate system. Camera calibration is a key step in computer vision for the $3 \mathrm{D}$ spatial information is extracted from the 2D images. 


\subsection{Stereo Rectification.}

When two image plane are completely aligned row, the computation of stereoscopic disparity is the simplest. Since two cameras are almost impossible to have an accurate coplanar and row aligned imaging plane, the perfect alignment structure almost does not exist in the real stereo system. The purpose of stereo correction is to reprojection of the image plane of the two cameras, so that they can be precisely on the same plane, and the line of the image is completely aligned to the front of the parallel structure.

\subsection{Stereo Matching.}

The 3D points of two different camera views are matched, and only the visual area of the overlap of the two cameras can be calculated, so the camera as close to the front as possible in parallel to facilitate better results. Once the physical coordinates of the camera or the size of the object in the scene are determined, the depth value can be obtained by measuring the disparity value of the triangle between the matching points in the two different camera views.

\subsection{Determination of Depth Information.}

When the disparity image is obtained by stereo matching, the depth image can be determined and the scene 3D information can be recovered. Generally speaking, the precision of distance measurement is proportional to the matching precision, and is inversely proportional to the length of the base line. The depth measurement accuracy can be improved by increasing the length of the baseline, but it can also the difference between the images and the difficulty of matching. Therefore, all aspects of the factors must be considered to stereo vision system can be accurate designed and each link with a high accuracy can be ensured.

\section{3D Depth of Field Acquisition Based on OpenCV}

The calibration method of Zhang Zheng you is used for the camera calibration, namely, the camera is aligned with the object of a lot of independent identifiable points(such as checkerboard). The relative position and orientation of the camera and the internal parameters of the camera can be calculated by viewing the object at different angles. This calibration method is not only simple and easy, but also with high calibration accuracy, so it has become one of the more commonly used calibration methods in the visual field. The $10^{*} 7$ checkerboard is used as the plane template in the design, and the size of each checkerboard is $27 * 27 \mathrm{~cm}$.

The calibration toolbox of MATLAB is used for camera calibration. The process of each image corner from the left camera is chosen in the calibration toolbox of MATLAB as shown in Figure 1; the relative position of the calibration board with respect to the camera coordinate system as shown in Figure 2.

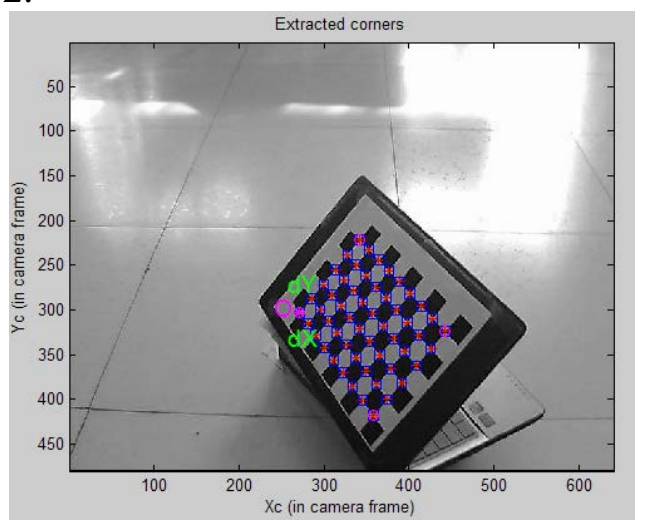

Fig. 1 The corner picking of left camera

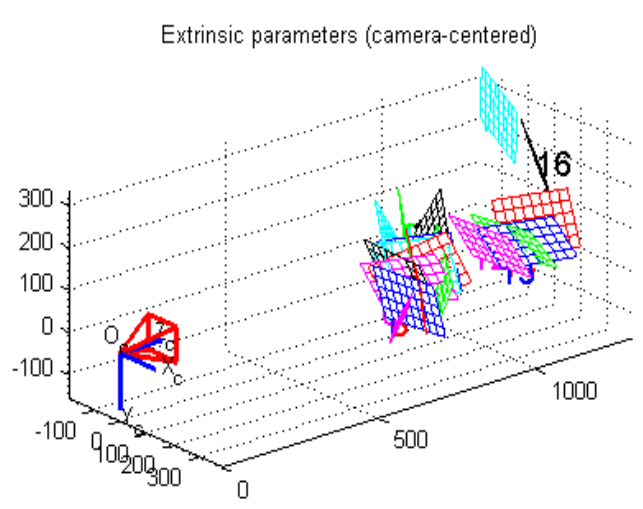

Fig. 2 The external parameters of left camera Calibration results of the intrinsic parameters in the left camera:

Focal Length: $\quad$ fc $=\left[\begin{array}{l}780.42072 \\ 778.12040\end{array}\right]$

Principal point: $\quad c c=\left[\begin{array}{ll}319.63967 & 266.47556\end{array}\right]$

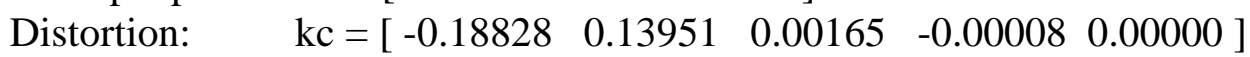


The same as calibration results of the intrinsic parameters in the right camera:

Focal Length: $\quad$ fc_right $=\left[\begin{array}{l}785.34228 \\ 784.01126\end{array}\right]$

Principal point: cc_right $=\left[\begin{array}{ll}322.29955252 .80320\end{array}\right]$

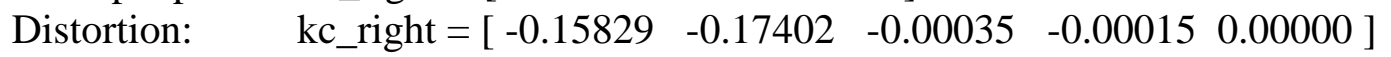

The stereo calibration of left and right camera is carried out by using the stereo_gui of Matlab Calibration Toolbox, the relative position of the calibration board with respect to the camera coordinate system and the relative position of the two cameras as shown in Figure 3.

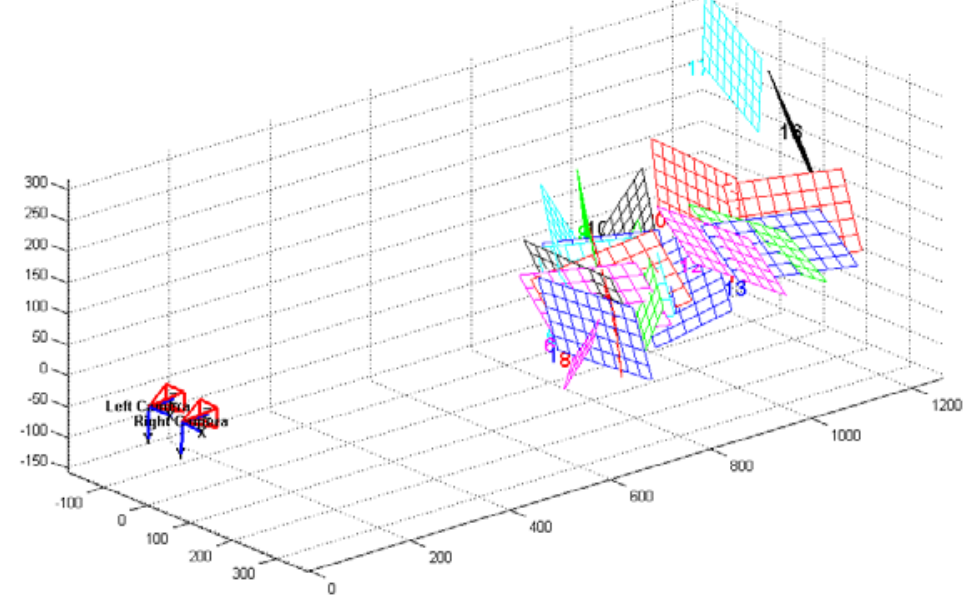

Fig. 3 Extrinsic parameters

The rotation and translation matrices of the two cameras are:

Rotation vector: $\quad$ om $=\left[\begin{array}{lll}-0.01850 & -0.03507 & -0.03742\end{array}\right]$

Translation vector: $\quad \mathrm{T}=\left[\begin{array}{lll}-80.62260 & 2.41138 & 0.49281\end{array}\right]$

After the stereo calibration of the left and right cameras, stereo rectificationis carried out by the calibration parameters, and the stereo matching is performed by using the corrected image. The purpose of stereo rectification: the reprojection of image plane of two cameras is on the same plane, and the line of the image is completely aligned to the front of the parallel structure, the distribution of the pole line in the normalized polar line constraint, which makes the matching efficiency further improved. Stereo matching can be carried out after the forward parallel alignment and line alignment of the left and right cameras' image, each row is a pole line after correction, so the matching position on the right image is bound to be in the same row of the left image, and only the visual area in the overlapping view of the two cameras can be calculated. Once we know the physical coordinates of the camera or the size of the object in the scene, we can get the depth value through the triangulation between the matching points in the two different camera views. The left and right camera images after stereo correction are shown in Figure 4.

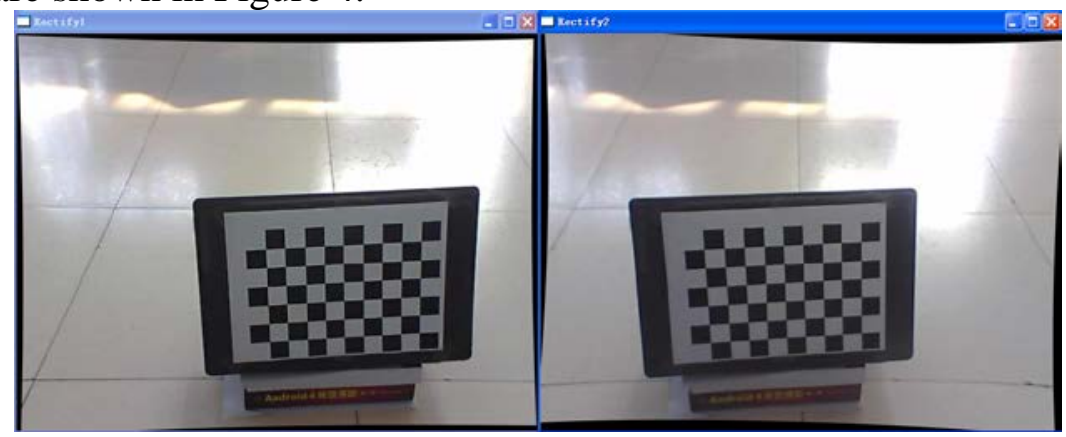

Fig. 4 Images of left and right camera after stereo rectification

\section{Experimental Result}

Based on acquisition principle of 3D depth of field and OpenCV function, the 3D depth of field acquisition system is developed by using vs2010, the dual camera parallel forward be placed, the depth of field of object is restored by the above five steps, program is verified to run stable after rigorous test. At the time of calibration, 20 photographs are taken by left and right camera, the inside 
and outside parameters of cameras are solved by using the corresponding function. Finally, one of a pair image of left and right camera is selected to solve the depth of field. The result of depth of field is shown in Figure 5.

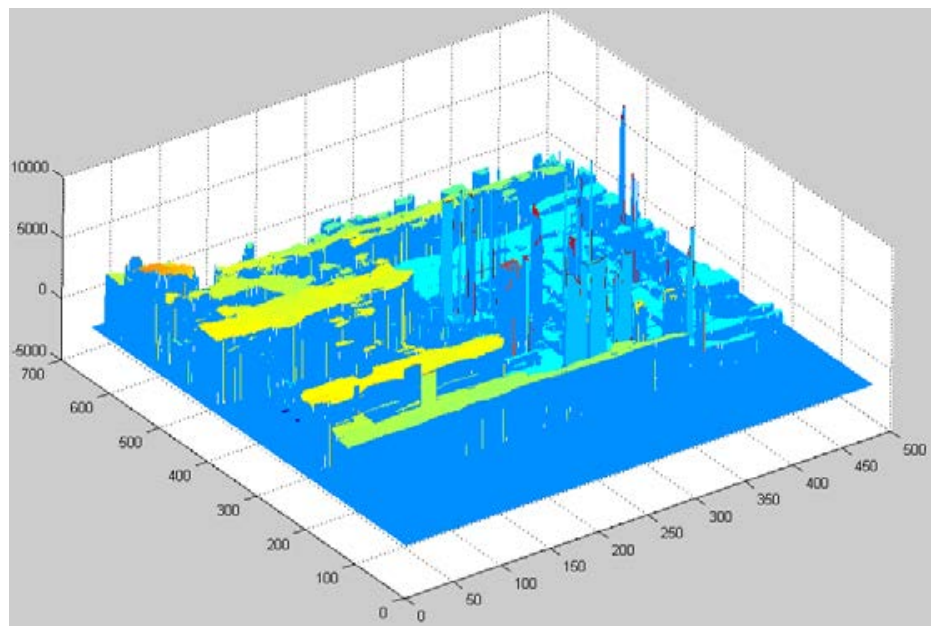

Fig. 5 The results of depth of field

\section{Summary}

In this paper, the 3D reconstruction system with the advantages of simple calculation, accurate results, high efficiency, cross platform and so on. The system can be effectively applied to all kinds of applications of computer vision. This test system is suitable to the 3D measurement that range is not too great and space object of less occlusion, the number of cameras need to be increased for more serious occlusion to take the objects from more directions, the 3D reconstruction is carried out by using vision principle of binocular stereo in multiple directions.

\section{Acknowledgments}

This work has been supported by the Technological Innovation Project of Jiamusi University Graduate (LM2014_012).

\section{References}

[1]. Park J S. Interactive 3D reconstruction from multiple images: a primitive-based approach. Pattern Recognition Letters. Vol. 26 (2005) No. 16, p. 2558-2571.

[2]. Zhang W, Gao X, Sung E et al. A feature-based matching scheme M PCD and robust matching strategy. Pattern Recognition Letters. Vol. 28 (2007) No. 16, p 1222-1231.

[3]. S. Birchfield and C. Tomasi. Multiway Cut for Stereo and Motion with Slanted Surfaces. Proceedings of the Seventh IEEE International Conference on Computer Vision. Greece, 1999, p. 489-495.

[4]. Kim H, Sohn K. Hierarchical disparity estimation with energy-based regularization. International Conference on Image Processing. 2003, p. 373-376.

[5]. Terzopoulos D. The Computation of Visible-Surface Representations. IEEE Transactions on Pattern Analysis and Machine Intelligence. 1988, 10 (4), p. 417-438.

[6]. Gyung-Bum Kim, Sung-Chong. An accurate and robust stereo matching algorithm with variable windows for 3D measurements. Mechatronics, 2004, p. 715-735. 